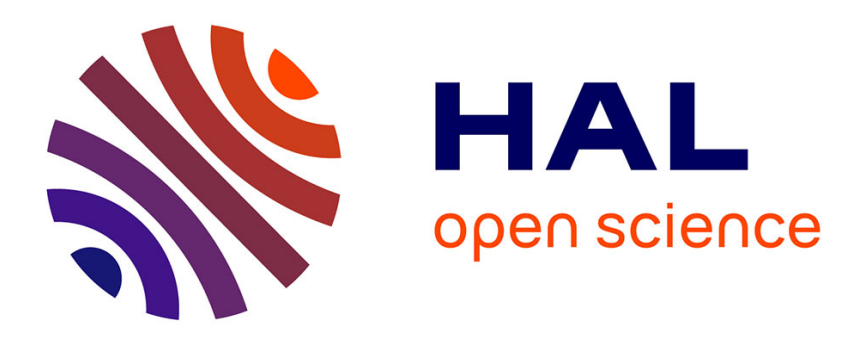

\title{
Classical electrodynamics of non-specular dielectric surfaces
}

\author{
F. García-Moliner, F. Flores
}

\section{To cite this version:}

F. García-Moliner, F. Flores. Classical electrodynamics of non-specular dielectric surfaces. Journal de Physique, 1977, 38 (7), pp.851-862. 10.1051/jphys:01977003807085100 . jpa-00208648

\section{HAL Id: jpa-00208648 https://hal.science/jpa-00208648}

Submitted on 1 Jan 1977

HAL is a multi-disciplinary open access archive for the deposit and dissemination of scientific research documents, whether they are published or not. The documents may come from teaching and research institutions in France or abroad, or from public or private research centers.
L'archive ouverte pluridisciplinaire HAL, est destinée au dépôt et à la diffusion de documents scientifiques de niveau recherche, publiés ou non, émanant des établissements d'enseignement et de recherche français ou étrangers, des laboratoires publics ou privés. 


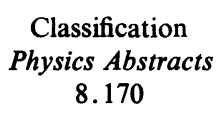

\title{
CLASSICAL ELECTRODYNAMICS OF NON-SPECULAR DIELECTRIC SURFACES
}

\author{
F. GARCÍA-MOLINER and F. FLORES \\ Instituto de Física del Estado Sólido (CSIC and UAM), \\ Universidad Autónoma, Cantoblanco, Madrid 34, Spain \\ (Reçu le 15 décembre 1976, révisé le 14 mars 1977, accepté le 18 mars 1977)
}

\begin{abstract}
Résumé. - L'effet de dispersion spatiale en électrodynamique des surfaces est étudié pour un modèle phénoménologique qui décrit la diffusion des excitations élémentaires sur la surface en termes d'un paramètre $p$, la surface spéculaire étant le cas $p=1$. L'impédance superficielle, la réflectivité et la relation de dispersion des modes de surface sont obtenues pour un $p$ arbitraire sans faire appel à des conditions de contours additionnelles. On montre que le problème est suffisamment défini si le modèle, l'effet de surface inclus, est spécifié. Plusieurs conditions de contours additionnelles utilisées dans la littérature sont discutées et caractérisées comme des modèles correspondant à des valeurs particulières de $p$. Les différents rôles des constantes diélectriques longitudinales et transversales sont discutés en termes physiques.
\end{abstract}

\begin{abstract}
The effect of spatial dispersion on the electrodynamics of surfaces is studied for a phenomenological model which describes the surface scattering of the elementary excitations in terms of a parameter $p$, the specular surface being the case $p=1$. Surface impedance, reflectivity and surface mode dispersion relation are obtained for arbitrary $p$ without resorting to additional boundary conditions. It is thus shown that the problem is sufficiently defined if the model, including the effect of the surface, is specified. Various forms of additional boundary conditions used in the literature are discussed and characterized as models corresponding to particular values of $p$. The different roles of the longitudinal and transverse dielectric constants are discussed in physical terms.
\end{abstract}

1. Introduction. - It is well known that the standard boundary conditions which result from imposing the correct matching behaviour of the EM field across a boundary do not contain sufficient information to solve the non-local form of Maxwell's equations for a semi-infinite dispersive medium whose dielectric function depends on wavevector $\mathbf{k}$ and frequency $\omega$. Agranovich and Ginzburg [1] pointed out that the problem is completely determined once the model has been defined. It is important to stress that this must include the effect of the surface on the scattering of the excitations of the system - e.g. excitons, polaritons, optical phonons or plasmons. Many authors [2-11] take a different view and formulate the problem in terms of additional boundary conditions (A.B.C.). Sometimes [4] the A.B.C. are simply postulated and then their consequences explored. Otherwise attempts are made at obtaining the A.B.C. from physical arguments about the behaviour of the electric polarization $\mathbf{P}$ and its normal derivative on crossing the surface $[2,3,6,8]$. A great deal of effort has been spent $[9,10,11]$ on producing formal arguments aimed at establishing the correct A.B.C. Briefly, the current situation is as follows : (i) It is often held that A.B.C. are necessary. (ii) It is sometimes implied that the problem of finding the correct A.B.C. is a meaningful question. (iii) Although it is often implicitly recognized that the A.B.C. in fact depend on the starting assumptions, there seems to be no readily available way of obtaining the A.B.C. as a function of some parameter characterizing the model, except for simple cases [9].

Consider an isotropic homogeneous medium with dielectric function $\varepsilon(\mathbf{k}, \omega)$. The assumption of isotropy is comparatively unimportant. The question of homogeneity is a more serious matter. It will not be touched upon in this paper, the aim of which is to provide a solution for the homogeneous case. It will be seen presently that in itself this is by no means a trivial problem if the surface is not specular. Now, the response of an isotropic medium is in general given by a tensor involving the two second rank tensors 
with full rotational symmetry, namely the dyad kk and the unit tensor $\mathbf{I}$. Thus the relationship between electric field $\mathbf{E}$ and displacement $\mathbf{D}$ is of the form

$$
\mathbf{D}=\varepsilon_{\mathrm{L}}(\mathbf{k}, \omega) \frac{\mathbf{k k}}{k^{2}} \cdot \mathbf{E}+\varepsilon_{\mathrm{T}}(\mathbf{k}, \omega)\left(\mathbf{I}-\frac{\mathbf{k k}}{k^{2}}\right) \cdot \mathbf{E}
$$

in Fourier transform. The longitudinal/transverse dielectric functions $\varepsilon_{\mathrm{L}} / \varepsilon_{\mathrm{T}}$ by definition relate $\mathbf{D}$ and $\mathbf{E}$ in the corresponding cases. Hence the dielectric tensor $\varepsilon$ is of the form

$$
\varepsilon=\left(\varepsilon_{\mathbf{L}}-\varepsilon_{\mathrm{T}}\right) \frac{\mathbf{k k}}{k^{2}}+\varepsilon_{\mathrm{T}} \mathbf{I}
$$

This very simple point will bear on the analysis later on.

Suppose the medium is contained in the half space $z>0$. Put $\mathbf{r}=(\boldsymbol{\rho}, z)$ in general, where $\boldsymbol{\rho}=(x, y)$ is the surface projection of $\mathbf{r}$. Then $\overline{\mathbf{r}}=(\boldsymbol{\rho},-z)$ is the mirror image of the point $\mathbf{r}$. At every point $r$ in $z>0$ there will be some field or excitation, say $D$, responding non locally to some stimulus, say $E$, everywhere else. The non-local integral can always be written in the form $(i, j=x, y, z)$

$$
\begin{aligned}
D_{i}(r)=\sum_{j} \int_{z^{\prime} \geqslant 0}\left[\varepsilon_{i j}\left(\mathbf{r}, \mathbf{r}^{\prime}\right) E_{j}\left(\mathbf{r}^{\prime}\right)+\right. & \\
& \left.+\varepsilon_{i j}\left(\mathbf{r}, \overline{\mathbf{r}}^{\prime}\right) E_{j}\left(\overline{\mathbf{r}}^{\prime}\right)\right] \mathrm{d} \mathbf{r}^{\prime} .
\end{aligned}
$$

This is merely an identical decomposition of the range of $z$. The model is introduced by assuming a fixed relationship between $E_{j}\left(\overline{\mathbf{r}}^{\prime}\right)$ and $E_{j}\left(\mathbf{r}^{\prime}\right)$. In a phenomenological way this can be done in terms of a parameter $p$. For example, if one writes

$$
E_{x}\left(\overline{\mathbf{r}}^{\prime}\right)=p E_{x}\left(\mathbf{r}^{\prime}\right),
$$

then $p=1$ is the specular surface model. The form of the relationship in terms of $p$ cannot be expressed in one single equation, applicable to all components of the field. These details will be set out in $\S 3$. Suffice it to say here that the model is completely specified by $\varepsilon_{\mathrm{L}}(\mathbf{k}, \omega), \varepsilon_{\mathrm{T}}(\mathbf{k}, \omega)$ and $p$, which can vary between 1 and -1 , corresponding to complete change of phase for the reflected wave. The purpose of this article is to show that the problem can be completely solved with just this information, without any reference to A.B.C., in agreement with Agranovich and Ginzburg [1].

Before doing this it may be useful to review the main attempts at obtaining the A.B.C., pointing out the assumptions therein contained. Thus Maradudin and Mills [2] assume that $\varepsilon$ is scalar which, after (1), means $\varepsilon_{\mathrm{L}}=\varepsilon_{\mathrm{T}}$. The implication of this will be discussed in $\S 4$. Furthermore, they assume

$$
\varepsilon\left(\mathbf{r}, \mathbf{r}^{\prime}\right)=\theta(z) \theta\left(z^{\prime}\right) \varepsilon\left(\mathbf{r}-\mathbf{r}^{\prime}\right),
$$

which amounts to assuming $p=0$, in which case the second term in the integrand in (2) vanishes. The particular behaviour they find for the component of $\mathbf{P}$ parallel to the surface is a direct consequence of these two assumptions, and not a generally valid relation. Birman and Zeyher [9] also assume the susceptibility $\chi$ is a scalar, i.e., $\chi_{\mathrm{L}}=\chi_{\mathrm{T}}$. Furthermore, they write for $\chi$ an expression of the form

$$
\begin{aligned}
\chi\left(\mathbf{r}, \mathbf{r}^{\prime}\right)=\chi_{0} \delta\left(\mathbf{r}-\mathbf{r}^{\prime}\right)+f_{1}\left(\left|\mathbf{r}-\mathbf{r}^{\prime}\right|\right)+ \\
+p f_{1}\left(\left|\mathbf{r}-\overline{\mathbf{r}}^{\prime}\right|\right) \theta(z) \theta\left(z^{\prime}\right)
\end{aligned}
$$

and obtain different A.B.C. for different values of $p$. Subsequently Frankel and Birman [10] use this approximation, with $p=-1$, to calculate the reflectivity and find appreciable disagreement with Maradudin and Mills [2], of course, since they use a different physical model. A different approach was taken by Skettrup [11] who constructed a Lagrangian. The claim is that the A.B.C. follow rigorously from the Weierstrass-Erdmann corner conditions. But it turns out [12] that the requirement for these to be valid is the continuity of the field variables, which in this case include the components of the polarization. Thus the behaviour of $\mathbf{P}$ is in fact postulated, and this amounts to choosing a model. In particular if $P_{x}=0$ for all $z \leqslant 0-$ in the vacuum - and $P_{x}$ is assumed to be continuous, then necessarily $P_{x}(+0)=0$. It will be seen in $\S 2$ that this corresponds to $p=-1$.

Now, solving the problem by explicitly using some A.B.C. which must first be found amounts only to adding an extra formal complication. It can be done, but it is not a general procedure as has often been maintained. Since all the arguments turn out to be based on some specific model, this does not add any further degree of rigour, in spite of formal appearance, and one might as well proceed directly from the model, which also gives a more transparent physical insight. This will be done in this paper for arbitrary $p$, maintaining in principle the difference between $\varepsilon_{\mathrm{T}}$ and $\varepsilon_{\mathrm{L}}$, finally making comparison with some of the representative results so far obtained using A.B.C.

2. The surface impedance and related properties. The problem can be formulated in the following way : (i) Given the half medium with dielectric functions $\varepsilon_{\mathrm{L}}$ and $\varepsilon_{\mathrm{T}}$, define a hypothetical extended medium (M) with the same $\varepsilon_{\mathrm{L}}$ and $\varepsilon_{\mathrm{T}}$ and also with some appropriate fictitious stimuli. These, which will eventually be eliminated, are by definition part of the hypothetical system (M). (ii) Calculate the field $\left(F^{M}\right)$ everywhere in (M). E.g., $F^{M}=E^{M}, H^{M}$, etc. (iii) Do likewise for the extended vacuum (V) and calculate $\mathbf{F}^{\mathbf{V}}$. Steps (i) and (iii) introduce some ad hoc subsidiary parameters describing the fictitious stimuli, which are later eliminated. (iv) Reconstitute the real system by matching $\mathbf{F}^{\mathrm{V}}(z<0)$ to $\mathbf{F}^{\mathrm{M}}(z>0)$ and imposing the correct behaviour that the real field must have at the boundary. This yields matching equations which involve the 
subsidiary parameters. (v) Eliminate these parameters by imposing fixed relationships between $\mathrm{F}^{\mathrm{M}}(z<0)$ and $F^{\mathrm{M}}(z>0)$. This is where the model is introduced in terms of the parameter $p$ to be used in (2).

The fictitious stimuli can be anything convenient, since they have no physical significance and are in the end eliminated. The following stimuli and corresponding response functions are introduced here for later use.

Electric current J. From Maxwell's equations, the response is, in Fourier transform according to $\exp (i(\mathbf{k . r}-\omega t))$

where

$$
\mathbf{E}(\mathbf{k}, \omega)=\mathbf{G}(\mathbf{k}, \omega) . \mathbf{J}(\mathbf{k}, \omega)
$$

$$
\begin{aligned}
\mathbf{G}(\mathbf{k}, \omega)=- & i \frac{4 \pi}{\omega} \frac{\mathbf{k} \mathbf{k}}{k^{2} \varepsilon_{\mathrm{L}}(\mathbf{k}, \omega)}+ \\
& +i \frac{4 \pi \omega}{c^{2}} \frac{\left(k^{2} \mathbf{I}-\mathbf{k k}\right)}{k^{2}\left(k^{2}-\omega^{2} \varepsilon_{\mathrm{T}}(\mathbf{k}, \omega) / c^{2}\right)}
\end{aligned}
$$

The dependence on $(\mathbf{r}, t)$ or $(\mathbf{k}, \omega)$ will be omitted henceforth whenever it can be easily understood.

Magnetic current $g$. If such a thing existed one would start from

$$
\nabla \Lambda \mathbf{E}=-\frac{1}{c} \frac{\partial \mathbf{H}}{\partial t}+\frac{4 \pi}{c} \mathbf{g} ; \quad \nabla \Lambda \mathbf{H}=\frac{1}{c} \frac{\partial \mathbf{D}}{\partial t},
$$

whence, in Fourier transform,

where

$$
\mathbf{H}=\mathbf{K} \cdot \mathbf{g},
$$

$$
\mathbf{K}(\mathbf{k}, \omega)=-i \frac{4 \pi \omega}{c^{2}} \frac{\left(\varepsilon_{\mathrm{T}} \mathbf{I}-c^{2} \mathbf{k k} / \omega^{2}\right)}{\left(k^{2}-\omega^{2} \varepsilon_{\mathrm{T}} / c^{2}\right)} .
$$

Notice that this response has also a longitudinal and a transverse part, but it only depends on $\varepsilon_{\mathrm{T}}$. This follows from (1) without further assumptions. What happens is simply that $\mathbf{H}$ has now non zero divergence.

The surface impedance can now be obtained for different models. From now on the wavevector will always be of the form $(\kappa, 0, \lambda)$, corresponding to angle of incidence $\theta$ and propagation direction $x$, i.e., $\kappa=k \sin \theta, \lambda=k \cos \theta$. Before carrying out a more elaborate analysis $-\S 3-$ it is convenient to consider here two extreme models of surface scattering.

(i) Specular or symmetric case $(p=1)$. For $P$-mode geometry, $\mathbf{E}=\left(E_{x}, 0, E_{z}\right), \mathbf{H}=\left(0, H_{y}, 0\right)$. Define an extended medium (M) with fictitious stimulus $\mathbf{J}^{\mathrm{M}}=\left(J_{\mathrm{s}}^{\mathrm{M}}, 0,0\right) \delta(z)$, i.e., $\left(J_{\mathrm{s}}^{\mathrm{M}} / 2 \pi, 0,0\right)$ in Fourier transform. This creates a field with mirror symmetry, in which

$$
E_{x}^{\mathrm{M}}(-z)=E_{x}^{\mathrm{M}}(+z) ; H_{y}^{\mathrm{M}}(-z)=-H_{y}^{\mathrm{M}}(+z) .
$$

Then

$$
E_{x}(+0)=\frac{J_{\mathrm{s}}^{\mathrm{M}}}{2 \pi} \lim _{\eta \rightarrow 0} \int \exp (i \lambda \eta) G_{x x} \mathrm{~d} \lambda \equiv \frac{J_{\mathrm{s}}^{\mathrm{M}}}{2 \pi} \mathcal{G}_{x x}^{(+)}
$$

Henceforth $\lim \eta \rightarrow 0$ will be understood wherever $\eta$ appears. The integrals are always from $-\infty$ to $+\infty$ and a script symbol like $\mathcal{G}$ indicates the corresponding surface projection of $G$. Since

$$
D_{x}^{\mathrm{M}}(-0)=D_{x}^{\mathrm{M}}(+0)=D_{x}(+0)
$$

and also $H_{y}^{\mathrm{M}}(+0)=H_{y}(+0), J_{\mathrm{s}}^{\mathrm{M}}$ is eliminated by integrating $\mathbf{\nabla} \Lambda \mathbf{H}^{\mathrm{M}}$ between $-\eta$ and $+\eta$, which yields

$$
J_{\mathbf{s}}^{\mathrm{M}}=-\frac{c}{2 \pi} H_{y}(+0) \text {. }
$$

From (7) and (8) the surface impedance is

$$
Z_{\mathrm{p}}=\frac{4 \pi}{c} \frac{E_{x}(+0)}{H_{y}(+0)}=-\frac{1}{\pi} \mathcal{G}_{x x}^{(+)} .
$$

For S-mode geometry, $\mathbf{E}=\left(0, E_{y}, 0\right)$,

$$
\mathbf{H}=\left(H_{x}, 0, H_{z}\right) .
$$

Use as fictitious stimulus $\mathbf{J}^{\mathbf{M}}=\left(0, J_{\mathrm{s}}^{\mathrm{M}}, 0\right) \delta(z)$. Then

$$
E_{y}(+0)=\frac{J_{s}^{\mathrm{M}}}{2 \pi} \mathcal{G}_{y y}^{(+)}
$$

and, integrating $\boldsymbol{\nabla} \Lambda \mathbf{H}^{\mathrm{M}}$ again,

$$
J_{\mathbf{s}}^{\mathrm{M}}=\frac{c}{2 \pi} H_{x}(+0) .
$$

The definition of the surface impedance is now different. Thus, from (10) and (11),

$$
Z_{\mathrm{s}}=-\frac{4 \pi}{c} \frac{E_{y}(+0)}{H_{x}(+0)}=-\frac{1}{\pi} \mathcal{G}_{y y}^{(+)}
$$

(ii) The antisymmetric case $(p=-1)$. This is obtained by using an appropriate $\mathbf{g}$ stimulus which produces a field in which it is $\mathbf{H}^{\mathbf{M}}$ that has mirror symmetry. For the P-mode it is sufficient to use $\left(0, g_{\mathrm{s}}^{\mathrm{M}}, 0\right) \delta(z)$. Then

$$
H_{y}(+0)=\frac{g_{\mathrm{s}}^{\mathrm{M}}}{2 \pi} \int \exp (i \lambda \eta) K_{y y} \mathrm{~d} \lambda \equiv \frac{g_{\mathrm{s}}^{\mathrm{M}}}{2 \pi} K_{y y}^{(+)} .
$$

Notice that this defines the surface projection $\mathcal{K}_{y y}^{(+)}$ of $K_{y y}$, much as eq. (7) defines $\mathcal{G}_{x x}^{(+)}$as surface projection of $G_{x x}$. Now, integrating $\nabla \Lambda \mathbf{E}^{\mathrm{M}}$,

$$
g_{\mathrm{s}}^{\mathrm{M}}=\frac{c}{2 \pi} E_{x}(+0)
$$

Hence

$$
\left(\frac{4 \pi}{c}\right)^{2} \frac{1}{Z_{\mathrm{p}}}=\frac{c}{4 \pi} \frac{H_{y}(+0)}{E_{x}(+0)}=\frac{1}{\pi} K_{y y}^{(+)} .
$$

For S-mode use $\left(g_{\mathrm{s}}^{\mathrm{M}}, 0,0\right) \delta(z)$. Then

$$
H_{x}(+0)=\frac{g_{\mathrm{s}}^{\mathrm{M}}}{2 \pi} \mathcal{K}_{x x}^{(+)},
$$


while

$$
g_{\mathrm{s}}^{\mathrm{M}}=-\frac{c}{2 \pi} E_{y}(+0) .
$$

Hence

$$
\left(\frac{4 \pi}{c}\right)^{2} \frac{1}{Z_{s}}=-\frac{c}{4 \pi} \frac{H_{x}(+0)}{E_{y}(+0)}=\frac{1}{\pi} \pi_{x x}^{(+)} .
$$

The explicit formulae for $Z$ are simply obtained by taking components in (4) or (6). This yields the formulae obtained by Kliewer and Fuchs [13] for $p=1$ and by Rimbey and Mahan [4] for $p=-1$. They clearly hold for different models of surface scattering, and for this reason neither one is more general than the other. Of course it is trivial to evaluate $P$ and to find that for, say, the P-mode, one has $\partial P_{x} / \partial z=0$ for $z=+0$ if $p=1$, and $P_{x}(+0)=0$ for $p=-1$, but the present derivation shows that this need not be invoked as A.B.C. In fact the final results have been obtained here without raising the question of A.B.C. Moreover, by focusing on the starting model for surface scattering one can clarify a point which seems to have led to some perplexity [14]. In a semiclassical theory involving electrons or free charge carriers the values of $p$ outside the range $(0,1)$ are physically meaningless. The case $p=1$ is the extreme ideal model, easiest to solve, and this is why it is mostly used. For dielectrics or excitonic systems $p$ can take meaningful values in the range $(-1,1)$. Here $p=-1$ is also an extreme ideal model, and it tends to be used rather often for this reason. Thus, there is a good reason why the condition $P_{x}(+0)=0$ is never used in the theory of surface plasmons. See the final discussion in $\S 5$.

Having thus framed the issue A.B.C. versus physical model, the discussion from now on will be concerned with the P-mode geometry, which is the physically interesting case in which $E$ has a component perpendicular to the surface and can therefore excite charge density waves in the medium. Now, if the surface impedances $Z, Z^{\prime}$ of two media are known, then the reflection at a plane separating these two media is [15]

$$
R=\left|\frac{Z^{\prime}-Z}{Z^{\prime}+Z}\right|^{2}
$$

In particular, the surface impedance of vacuum is

$$
Z^{\prime}=\frac{4 \pi}{c} \cos \theta
$$

This follows from simple geometry or else it can be obtained from (9), (12), (13) or (14), indistinctly, putting $\varepsilon_{\mathrm{L}}=\varepsilon_{\mathrm{T}}=1$. Thus, for the $\mathrm{V} / \mathrm{M}$ surface,

$$
R=\left|\frac{\cos \theta-\frac{c}{4 \pi} Z}{\cos \theta+\frac{c}{4 \pi} Z}\right|^{2}=\left|\frac{\frac{4 \pi}{c} \cos \theta-Z}{\frac{4 \pi}{c} \cos \theta+Z}\right|^{2} \text {. }
$$

Now, the reflectivity is a response function. Its poles yield the surface modes. Thus, putting

$$
\cos \theta=\left(1-\frac{c^{2} \kappa^{2}}{\omega^{2}}\right)^{1 / 2}
$$

the surface mode dispersion relation (S.M.D.R.) is

$$
\frac{4 \pi}{c}\left(1-\frac{c^{2} \kappa^{2}}{\omega^{2}}\right)^{1 / 2}+Z(\kappa, \omega)=0 .
$$

Therefore it suffices to find $Z$. Then one knows $R$ and the S.M.D.R. For example, surface plasmons for inhomogeneous space charge layers in semiconductors have been obtained in this way [16]. Vice versa, if one knows, say, the S.M.D.R. in the form $f(\kappa, \omega)=0$, then one has

$$
Z=f(\kappa, \omega)-\frac{4 \pi}{c}\left(1-\frac{c^{2} \kappa^{2}}{\omega^{2}}\right)^{1 / 2}
$$

and hence $R$. This could be wrong by some unknown non zero factor, i.e., if the actual dispersion relation happens to be some factor $\alpha$ times the 1.h.s. of (15), then (16) would not give $Z$, but $\cos \theta(\alpha-1)+Z \alpha$. This is easily sorted out since $Z$ has been found explicitly for the easy cases $p=1$ and $p=-1$. Either one can be used to find $\alpha$ and thus resolve the possible ambiguity. Therefore, it suffices to solve any one of the three problems, $Z, R$ or S.M.D.R. for arbitrary $p$ and one knows the answer for the three together. This point tends to be overlooked and often leads to redundant effort. The rest of this article will deal with the S.M.D.R. for arbitrary $p$, in which case only homogeneous equations are involved and inessential factors can be omitted.

\section{Surface mode dispersion relation for arbitrary $p$.} - The approach outlined in $\S 2$ will now be used to study the S.M.D.R. for arbitrary $p$. The real field must have correct behaviour at the boundary. This yields the two matching equations

and

$$
\begin{aligned}
& H_{y}^{\mathrm{V}}(-0)=H_{y}^{\mathrm{M}}(+0) \\
& E_{x}^{\mathrm{V}}(-0)=E_{x}^{\mathrm{M}}(+0) .
\end{aligned}
$$

The fields in the extended medium must have the behaviour corresponding to the choice of model. This yields the subsidiary equations

$$
\begin{gathered}
\nabla \cdot \mathbf{E}^{\mathrm{M}}(z<0)=p \nabla \cdot \mathbf{E}^{\mathrm{M}} \quad(z>0), \\
\nabla \Lambda \mathbf{E}^{\mathrm{M}}(z<0)=-p \nabla \Lambda \mathbf{E}^{\mathrm{M}} \quad(z>0) \\
E_{x}^{\mathrm{M}}(-0)=p E_{x}^{\mathrm{M}}(+0) .
\end{gathered}
$$

The last equation is necessary because the divergence and the curl do not uniquely determine $\mathbf{E}$. This behaviour can be ensured by using as ficticious stimuli 
a suitable distribution of electric and magnetic currents, which in general may have a surface and a volume part, provided the latter is zero in $z>0$, where $\mathbf{E}^{\mathrm{M}}$ and $\mathbf{H}^{\mathrm{M}}$ must be equal to $\mathbf{E}$ and $\mathbf{H}$ of the real system. Then, in Fourier transform,

$$
\mathbf{J}^{\mathbf{M}}=\mathbf{J}_{\mathbf{s}}^{\mathbf{M}}(\kappa)+\mathbf{J}_{\mathbf{b}}^{\mathbf{M}}(\kappa, \lambda) .
$$

For the P-mode

$$
\mathbf{J}_{\mathbf{s}}^{\mathrm{M}}=\left(J_{\mathrm{s}}^{\mathrm{M}}, 0,0\right) ; \quad \mathbf{J}_{\mathrm{b}}^{\mathrm{M}}=\left(J_{\mathrm{bx}}, 0, J_{\mathrm{bz}}\right)
$$

Put

$$
J_{\mathrm{bx}}^{\mathrm{M}}+\frac{\lambda}{\kappa} J_{\mathrm{bz}}^{\mathrm{M}}=f_{1} J_{\mathrm{s}}^{\mathrm{M}} ; \frac{\lambda}{\kappa} J_{\mathrm{bx}}^{\mathrm{M}}-J_{\mathrm{bz}}^{\mathrm{M}}=f_{2} J_{\mathrm{s}}^{\mathrm{M}} .
$$

Then, with $j=\kappa J_{\mathrm{s}}^{\mathrm{M}}$,

$$
\mathbf{k} \cdot \mathbf{J}^{\mathrm{M}}=j\left(1+f_{1}\right) ; \quad \mathbf{k} \Lambda \mathbf{J}^{\mathrm{M}}=j\left(f_{2}+\frac{\lambda}{\kappa}\right) \mathbf{y}^{\mathbf{0}} .
$$

For $\mathbf{g}^{\mathrm{M}}$ it suffices to take - for the P-mode :

$$
\mathbf{g}_{\mathrm{s}}^{\mathrm{M}}=\left(0, g_{\mathrm{s}}^{\mathrm{M}}, 0\right) ; \quad \mathbf{g}_{\mathrm{b}}^{\mathrm{M}}=\left(0, g_{\mathrm{b}}^{\mathrm{M}}, 0\right) .
$$

Then the only component of $\mathbf{g}_{\mathrm{s}}^{\mathrm{M}}$ is, in Fourier transform,

$$
\begin{aligned}
& g_{\mathbf{s}}^{\mathbf{M}}(\kappa)+g_{\mathrm{b}}^{\mathrm{M}}(\kappa, \lambda)=g_{\mathrm{s}}^{\mathrm{M}}(\kappa)\left[1+\frac{g_{\mathrm{b}}^{\mathrm{M}}(\kappa, \lambda)}{g_{\mathrm{s}}^{\mathrm{M}}(\kappa)}\right] \equiv \\
& \equiv g_{\mathrm{s}}\left(1+f_{3}\right) .
\end{aligned}
$$

Now, the complete fields in (M) are

$$
\mathbf{E}^{\mathrm{M}}=\mathbf{E}^{\mathrm{MJ}}+\mathbf{E}^{\mathrm{M} g} ; \mathbf{H}^{\mathrm{M}}=\mathbf{H}^{\mathrm{MJ}}+\mathbf{H}^{\mathrm{M} \boldsymbol{\theta}},
$$

where

$$
\begin{aligned}
\mathbf{E}^{\mathrm{MJ}}=\{- & i \frac{4 \pi}{\omega} \frac{\mathbf{k} \mathbf{k}}{k^{2} \varepsilon_{\mathrm{L}}}+ \\
& \left.+i \frac{4 \pi \omega}{c^{2}} \frac{\left(k^{2} \mathbf{I}-\mathbf{k k}\right)}{k^{2}\left(k^{2}-\frac{\omega^{2}}{c^{2}} \varepsilon_{\mathrm{T}}\right)}\right\} \cdot \mathbf{J}^{\mathrm{M}}, \\
\mathbf{E}^{\mathrm{M} g} & =i \frac{4 \pi}{c} \frac{\mathbf{k} \Lambda \mathbf{g}^{\mathrm{M}}}{k^{2}-\frac{\omega^{2}}{c^{2}} \varepsilon_{\mathrm{T}}} \\
\mathbf{H}^{\mathrm{M} J} & =i \frac{4 \pi}{c} \frac{\mathbf{k} \Lambda \mathbf{J}^{\mathrm{M}}}{k^{2}-\frac{\omega^{2}}{c^{2}} \varepsilon_{\mathrm{T}}}
\end{aligned}
$$

and

$$
\mathbf{H}^{\mathrm{M} g}=-i \frac{4 \pi \omega}{c^{2}} \frac{\varepsilon_{\mathrm{T}}}{k^{2}-\frac{\omega^{2}}{c^{2}} \varepsilon_{\mathrm{T}}} \mathbf{g}^{\mathrm{M}} .
$$

Both (23) and (24) are to be used in these equations, and also in (19) and (20).

For the extended vacuum (V) it is sufficient to put a fictitious stimulus $J^{\mathbf{V}}=\left(J^{\mathbf{v}}, 0,0\right) \delta(z)$, which produces the corresponding $\mathbf{E}^{\mathrm{VJ}}$ and $\mathbf{H}^{\mathrm{VJ}}$.

Introducing the parameters

$$
\zeta=\frac{i J^{\mathrm{v}}}{j}=\frac{i J^{\mathrm{v}}}{\kappa J_{\mathrm{s}}^{\mathrm{M}}} ; \quad \xi=-i \frac{\omega}{c} \frac{g_{\mathrm{s}}}{j}=-i \frac{\omega}{c} \frac{g_{\mathrm{s}}}{\kappa J_{\mathrm{s}}^{\mathrm{M}}}
$$

the matching equations after (17) and (18) are :

$$
\begin{gathered}
\zeta \int \frac{\exp (-i \lambda \eta) \lambda \mathrm{d} \lambda}{k^{2}-\frac{\omega^{2}}{c^{2}}}=\xi \int \frac{\exp (i \lambda \eta) \varepsilon_{\mathrm{T}}\left(1+f_{3}\right)}{k^{2}-\frac{\omega^{2}}{c^{2}} \varepsilon_{\mathrm{T}}} \mathrm{d} \lambda+i \int \frac{\exp (i \lambda \eta)\left(f_{2}+\frac{\lambda}{\kappa}\right) \mathrm{d} \lambda}{k^{2}-\frac{\omega^{2}}{c^{2}} \varepsilon_{\mathrm{T}}} \\
\zeta\left\{-\kappa^{2} \int \frac{\exp (i \lambda \eta) \mathrm{d} \lambda}{k^{2}}+\frac{\omega^{2}}{c^{2}} \int \frac{\exp (-i \lambda \eta) \lambda^{2} \mathrm{~d} \lambda}{k^{2}\left(k^{2}-\frac{\omega^{2}}{c^{2}}\right)}\right\}=\xi \int \frac{\exp (i \lambda \eta) \lambda\left(1+f_{3}\right)}{k^{2}-\frac{\omega^{2}}{c^{2}} \varepsilon_{\mathrm{T}}} \mathrm{d} \lambda+ \\
+i\left\{-\kappa \int \frac{\exp (i \lambda \eta)\left(1+f_{1}\right) \mathrm{d} \lambda}{k^{2} \varepsilon_{\mathrm{L}}}+\frac{\omega^{2}}{c^{2}} \int \frac{\exp (i \lambda \eta) \lambda\left(f_{2}+\frac{\lambda}{\kappa}\right)}{k^{2}\left(k^{2}-\frac{\omega^{2}}{c^{2}} \varepsilon_{\mathrm{T}}\right)} \mathrm{d} \lambda\right\} .
\end{gathered}
$$

Eliminating $\zeta$ this yields the general form of the S.M.D.R.

$$
\begin{aligned}
& i \kappa \int \frac{\exp (i \lambda \eta)\left(1+f_{1}\right)}{k^{2} \varepsilon_{\mathrm{L}}} \mathrm{d} \lambda=\xi \int \frac{\exp (i \lambda \eta)\left(i v \varepsilon_{\mathrm{T}}+\lambda\right)\left(1+f_{3}\right)}{k^{2}-\frac{\omega^{2}}{c^{2}} \varepsilon_{\mathrm{T}}} \mathrm{d} \lambda+ \\
& +\int \frac{\exp (i \lambda \eta)\left(i \frac{\omega^{2}}{c^{2}} \frac{\lambda}{k^{2}}-v\right)\left(f_{2}+\frac{\lambda}{\kappa}\right)}{k^{2}-\frac{\omega^{2}}{c^{2}} \varepsilon_{\mathrm{T}}} \mathrm{d} \lambda,
\end{aligned}
$$


where $v^{2}=\kappa^{2}-\frac{\omega^{2}}{c^{2}}$. For later reference, notice that this can be written in the form

$$
i \kappa \int \frac{\exp (i \lambda \eta)\left(1+f_{1}\right)}{k^{2}}\left(\frac{1}{\varepsilon_{\mathrm{L}}}+1\right) \mathrm{d} \lambda=\xi \int \frac{\exp (i \lambda \eta)\left(i v \varepsilon_{\mathrm{T}}+\lambda\right)\left(1+f_{3}\right)}{k^{2}-\frac{\omega^{2}}{c^{2}} \varepsilon_{\mathrm{T}}} \mathrm{d} \lambda+Q,
$$

where

$$
Q=\int \exp (i \lambda \eta) \frac{\left(i \frac{\omega^{2}}{c^{2}} \lambda-v\right)\left(f_{2}+\frac{\lambda}{\kappa}\right)}{k^{2}-\frac{\omega^{2}}{c^{2}} \varepsilon_{\mathrm{T}}} \mathrm{d} \lambda+i \kappa \int \exp (i \lambda \eta) \frac{\left(1+f_{1}\right) \mathrm{d} \lambda}{k^{2}} .
$$

In the quasi-static $c \rightarrow \infty, v \rightarrow \kappa$ and $Q$ becomes

$$
Q_{\infty}=i \kappa \int \frac{\exp (i \lambda \eta)\left(f_{1}+i f_{2}\right)}{k^{2}} \mathrm{~d} \lambda .
$$

Now, from (22),

$$
J_{\mathrm{bx}}^{\mathrm{M}}(\kappa, \lambda)=\frac{\kappa^{2}}{k^{2}}\left(f_{1}+\frac{\lambda}{\kappa} f_{2}\right) J_{\mathrm{s}}^{\mathrm{M}}
$$

and in real space $J_{\mathrm{bx}}^{\mathrm{M}}(\kappa, z)$ is zero for $z>0$. Hence $Q_{\infty}$ vanishes identically and the S.M.D.R. is, in the quasistatic limit,

$$
i \kappa \int \exp (i \lambda \eta) \frac{\left(1+f_{1}\right)}{k^{2}}\left(\frac{1}{\varepsilon_{\mathrm{L}}}+1\right) \mathrm{d} \lambda=\xi \int \frac{\exp (i \lambda \eta)\left(i \kappa \varepsilon_{\mathrm{T}}+\lambda\right)\left(1+f_{3}\right)}{k^{2}} \mathrm{~d} \lambda .
$$

So far $f_{1}, f_{2}, f_{3}$ and $\xi$ are still unknown, except for the condition that $f_{1}, f_{2}$ and $f_{3}$ must not have poles in the upper half plane - complex $\lambda$ plane - in order to ensure that they vanish in the positive $z$ half space. The next question is to investigate the subsidiary conditions. These will first be written in general form and then evaluated for a specific model of the bulk medium. Following common practice, the model is defined by taking the following forms for $\varepsilon_{\mathrm{L}}$ and $\varepsilon_{\mathrm{T}}$ :

$$
\varepsilon_{\mathrm{L}}=\varepsilon_{\mathrm{c}}+\frac{\omega_{\mathrm{p}}^{2}}{\omega_{\mathrm{T}}^{2}-\omega^{2}+\beta_{\mathrm{L}}^{2} k^{2}}=\varepsilon_{\mathrm{c}}\left(1+\frac{\Omega_{\mathrm{p}}^{2}}{\omega_{\mathrm{T}}^{2}-\omega^{2}+\beta_{\mathrm{L}}^{2} k^{2}}\right)
$$

i.e.,

where

$$
\varepsilon_{\mathrm{L}}=\varepsilon_{\mathrm{c}} \frac{(\lambda+i L)(\lambda-i L)}{(\lambda+i l)(\lambda-i l)}
$$

$$
L=\sqrt{\kappa^{2}+\frac{\omega_{\mathrm{T}}^{2}+\Omega_{\mathrm{p}}^{2}-\omega^{2}}{\beta_{\mathrm{L}}^{2}}} ; \quad l=\sqrt{\kappa^{2}+\frac{\omega_{\mathrm{T}}^{2}-\omega^{2}}{\beta_{\mathrm{L}}^{2}}} .
$$

Also

$$
\varepsilon_{\mathrm{T}}=\varepsilon_{\mathrm{c}}\left(1+\frac{\Omega_{\mathrm{p}}^{2}}{\omega_{\mathrm{T}}^{2}-\omega^{2}+\beta_{\mathrm{T}}^{2} k^{2}}\right)
$$

i.e.,

$$
\varepsilon_{\mathrm{T}}=\varepsilon_{\mathrm{c}} \frac{(\lambda+i T)(\lambda-i T)}{(\lambda+i t)(\lambda-i t)}
$$

where

$$
T=\sqrt{\kappa^{2}+\frac{\omega_{\mathrm{T}}^{2}+\Omega_{\mathrm{p}}^{2}-\omega^{2}}{\beta_{\mathrm{T}}^{2}}} ; \quad t=\sqrt{\kappa^{2}+\frac{\omega_{\mathrm{T}}^{2}-\omega^{2}}{\beta_{\mathrm{T}}^{2}}} .
$$

Then

$$
\frac{1}{k^{2}-\frac{\omega^{2}}{c^{2}} \varepsilon_{\mathrm{T}}}=\frac{(\lambda+i t)(\lambda-i t)}{(\lambda+i R)(\lambda-i R)(\lambda+i r)(\lambda-i r)},
$$


with

$$
\begin{aligned}
& R^{2}=\frac{\left(\kappa^{2}+t^{2}-\varepsilon_{\mathrm{c}} \frac{\omega^{2}}{c^{2}}\right)+\sqrt{\left(\kappa^{2}+t^{2}-\varepsilon_{\mathrm{c}} \frac{\omega^{2}}{c^{2}}\right)^{2}-4\left(\kappa^{2} t^{2}-\varepsilon_{\mathrm{c}} \frac{\omega^{2}}{c^{2}} T^{2}\right)}}{2} \\
& r^{2}=\frac{\left(\kappa^{2}+t^{2}-\varepsilon_{\mathrm{c}} \frac{\omega^{2}}{c^{2}}\right)-\sqrt{\left(\kappa^{2}+t^{2}-\varepsilon_{\mathrm{c}} \frac{\omega^{2}}{c^{2}}\right)^{2}-4\left(\kappa^{2} t^{2}-\varepsilon_{\mathrm{c}} \frac{\omega^{2}}{c^{2}} T^{2}\right)}}{2} .
\end{aligned}
$$

These formulae will be used frequently in the rest of the paper. One can now see the form that $f_{1}, f_{2}$ and $f_{3}$ must take as functions of $\lambda$, for given $(\kappa, \omega)$, by using the subsidiary conditions. From (23), the general form of (19) is

$$
\int \exp (-i \lambda z) \frac{\left(1+f_{1}\right)}{\varepsilon_{\mathrm{L}}} \mathrm{d} \lambda=p \int \exp (i \lambda z) \frac{\left(1+f_{1}\right)}{\varepsilon_{\mathrm{L}}} \mathrm{d} \lambda
$$

From (35), the only dependence of the r.h.s. of (39) on $z$ is in the term $\exp (-L z)$, coming from the pole at $\lambda=i L$ when the integration contour is closed through the upper half circle at infinity. Thus, on closing the contour through the lower half circle for the 1.h.s., there will be no extra residue from $\left(1+f_{1}\right)$ if $f_{1}$ has the form

$$
f_{1}=\frac{B_{0}}{\lambda+i l}
$$

in which case the factor $(\lambda+i l)$ is cancelled by the same factor in $\varepsilon_{\mathrm{L}}$. This leaves $B_{0}$, in general a function of $\kappa$ and $\omega$, as a subsidiary parameter obeying the equation

$$
1+\frac{B_{0}}{i(l-L)}=p\left[1+\frac{B_{0}}{i(l+L)}\right]
$$

which results from evaluating (39) for the said model dielectric function.

Likewise for condition (20) it is necessary to form $\mathbf{k} \Lambda \mathbf{E}^{\mathrm{M}}$. After (25), (26) and (23) :

$$
\frac{\omega}{i 4 \pi} \mathbf{k} \Lambda \mathbf{E}^{\mathrm{M}}=-\frac{\omega}{c} g_{\mathrm{s}} \frac{k^{2}\left(1+f_{3}\right)}{k^{2}-\frac{\omega^{2}}{c^{2}} \varepsilon_{\mathrm{T}}}+\frac{\omega^{2}}{c^{2}} j \frac{\left(f_{2}+\frac{\lambda}{\kappa}\right)}{k^{2} \cdot-\frac{\omega^{2}}{c^{2}} \varepsilon_{\mathrm{T}}} .
$$

This yields a second subsidiary condition in a general form rather like (39), but with the more complicated structure of $\mathbf{k} \Lambda \mathbf{E}^{\mathrm{M}}$. The form of $f_{2}$ is obtained from a similar, though somewhat more involved, argument. It is clear that $f_{2}$ must contain a factor $(\lambda+i t)$ in the denominator, in order to cancel the same factor in (38), but its structure needs further analysis. From (22), both $J_{\mathbf{b x}}^{\mathrm{M}}$ and $J_{\mathbf{b z z}}^{\mathrm{M}}$ will be combinations of $(\lambda+i l)^{-1}$ and $(\lambda+i t)^{-1}$. Moreover, $J_{b x}^{\mathrm{M}}$ will contain a constant term, associated with the fact that $J_{\mathbf{s}}^{\mathrm{M}}$ is in the $x$ direction. Thus they will be of the general form

$$
\begin{aligned}
& J_{\mathrm{b} x}^{\mathrm{M}}=\left(\frac{A_{1}}{\lambda+i l}+\frac{A_{2}}{\lambda+i t}-B_{1}\right)^{\prime} J_{\mathrm{s}}^{\mathrm{M}}, \\
& J_{\mathrm{bz}}^{\mathrm{M}}=\left(\frac{A_{3}}{\lambda+i l}+\frac{A_{4}}{\lambda+i t}\right) J_{\mathrm{s}}^{\mathrm{M}} .
\end{aligned}
$$

Forming now the two combinations (22) and setting equal to zero the coefficient of $(\lambda+i t)^{-1}$ in $f_{1}$ and of $(\lambda+i l)^{-1}$ in $f_{2}$, yields

$$
A_{4}=\frac{\kappa}{i t} A_{2} ; \quad A_{1}=-\frac{\kappa}{i l} A_{3} .
$$

Thus there are only two independent parameters. This yields

$$
f_{1}=\frac{\left(l^{2}-\kappa^{2}\right)}{i l \kappa} \frac{A_{3}}{\lambda+i l}+\left(\frac{A_{3}}{\kappa}+\frac{A_{2}}{i t}-B_{1}\right)
$$

and

$$
f_{2}=-\frac{\lambda}{\kappa} B_{1}+\left(\frac{A_{2}}{\kappa}-\frac{A_{3}}{i l}\right)+\frac{\left(t^{2}-\kappa^{2}\right)}{i t \kappa} \frac{A_{2}}{\lambda+i t} .
$$

Hence $f_{2}$ has the form

$$
f_{2}=-\frac{\lambda}{\kappa} B_{1}+B_{2}+\frac{B_{3}}{\lambda+i t},
$$


with

$$
\begin{aligned}
& B_{0}=\frac{\left(l^{2}-\kappa^{2}\right)}{i l \kappa} A_{3} \\
& B_{1}=\frac{1}{\kappa} A_{3}+\frac{1}{i t} A_{2} \\
& B_{2}=-\frac{A_{3}}{i l}+\frac{A_{2}}{\kappa} \\
& B_{3}=\frac{\left(t^{2}-\kappa^{2}\right)}{i t \kappa} A_{2} .
\end{aligned}
$$

Eliminating $A_{2}$ and $A_{3}$ :

$$
\begin{aligned}
& B_{1}=\frac{i l}{\left(l^{2}-\kappa^{2}\right)} B_{0}+\frac{\kappa}{\left(t^{2}-\kappa^{2}\right)} B_{3} \\
& B_{2}=-\frac{\kappa}{\left(l^{2}-\kappa^{2}\right)} B_{0}+\frac{i t}{\left(t^{2}-\kappa^{2}\right)} B_{3} .
\end{aligned}
$$

This is to be used in the second subsidiary condition, which also contains the term in $k^{2}\left(1+f_{3}\right)$. On closing the contour of integration in the lower half plane, it is seen that $f_{3}$ needs a factor $(\lambda+i \kappa)^{-1}$, which is cancelled by $(\lambda+i \kappa)$ in $k^{2}=\lambda^{2}+\kappa^{2}$. This leads to

$$
f_{3}=\frac{B_{4}}{\lambda+i \kappa}
$$

Thus, besides $B_{0}$ which is given by (41), there are another three unknown parameters, namely $\xi, B_{3}$ and $B_{4}$. Hence three more equations are needed. Two of these come from the second subsidiary condition (20) in the following way. The $z$ dependence comes from the poles at $\lambda= \pm i r$ and $\lambda= \pm i R$. This yields the two functions $\exp (-r|z|)$ and $\exp (-R|z|)$ whose coefficients must independently obey (20). This yields

$$
\begin{aligned}
\left(\kappa^{2}-r^{2}\right) g_{\mathrm{s}}\{(1+p)+( & \left.\left.\frac{1}{\kappa-r}+\frac{p}{\kappa+r}\right) \frac{B_{4}}{i}\right\}- \\
& -\frac{\omega}{c} j\left\{(1+p) B_{2}+\frac{(p-1) i r}{\kappa}\left(1-B_{1}\right)+\left(\frac{1}{t-r}+\frac{p}{t+r}\right) \frac{B_{3}}{i}\right\}=0
\end{aligned}
$$

and

$$
\begin{aligned}
\left(\kappa^{2}-R^{2}\right) g_{\mathrm{s}}\{(1+p)+ & \left.\left(\frac{1}{\kappa-R}+\frac{p}{\kappa+R}\right) \frac{B_{4}}{i}\right\}- \\
& -\frac{\omega}{c} j\left\{(1+p) B_{2}+\frac{(p-1) i R}{\kappa}\left(1-B_{1}\right)+\left(\frac{1}{t-R}+\frac{p}{t+R}\right) \frac{B_{3}}{i}\right\}=0 .
\end{aligned}
$$

Finally, the last subsidiary condition is, after (21) :

$$
\int(\exp (-i \lambda \eta)-p \exp (i \lambda \eta)) E_{x}^{\mathrm{M}}(\kappa, \lambda) \mathrm{d} \lambda=0,
$$

where $E_{x}^{\mathrm{M}}$ is obtained from (25), (26), (23) and (24). On performing the integrations for the model dielectric functions (34) and (36), there are contributions from the poles at $\lambda= \pm i r, \pm i R, \pm i L$ and also at $\lambda= \pm i \kappa$, giving the corresponding exponentials. The coefficients of the first three vanish identically because of (45), (46) and (41), respectively. The vanishing of the coefficient of $\exp (-\kappa|z|)$ yields the last subsidiary condition :

$$
2 \frac{c}{\omega} i \kappa B_{4} g_{\mathrm{s}}=j\left\{\frac{\left(1+f_{1}(-i \kappa)\right)}{i}-f_{2}(-i \kappa)+i\right\}+p j\left\{\frac{\left(1+f_{1}(i \kappa)\right)}{i}+f_{2}(i \kappa)+i\right\}
$$

from which $B_{4}$ can be found. In fact, using (40), (42) and (43) in (48) yields

$$
B_{4}=0 \text {. }
$$

Thus, although the volume part of the fictitious stimulus $\mathbf{g}^{\mathrm{M}}$ is needed in the formal argument to guarantee the desired behaviour of the complete EM field in (M), in actual fact it turns out to vanish for arbitrary $p$. This simplifies a great deal the S.M.D.R. (32), in which $f_{3}=0$, and also the subsidiary conditions (45) and (46), which now become

$$
\begin{aligned}
(p+1)\left(r^{2}-\kappa^{2}\right) \xi=i \frac{\omega^{2}}{c^{2}}\left\{\frac{(p-1) i r}{\kappa}+\left(\frac{(p-1) l r}{\kappa}-\right.\right. & (p+1) \kappa) \frac{B_{0}}{l^{2}-\kappa^{2}}+ \\
& \left.+\frac{i\left(\kappa^{2}-r^{2}\right)[(1+p) t+(1-p) r]}{\left(t^{2}-\kappa^{2}\right)\left(t^{2}-r^{2}\right)} B_{3}\right\}
\end{aligned}
$$


and

$$
\begin{aligned}
(p+1)\left(R^{2}-\kappa^{2}\right) \xi=i \frac{\omega^{2}}{c^{2}}\left\{\frac{(p-1) i R}{\kappa}+\left(\frac{(p-1) l R}{\kappa}-(p+1) \kappa\right) \frac{B_{0}}{l^{2}-\kappa^{2}}+\right. & \\
& \left.+\frac{i\left(\kappa^{2}-R^{2}\right)[(1+p) t+(1-p) R]}{\left(t^{2}-\kappa^{2}\right)\left(t^{2}-R^{2}\right)} B_{3}\right\} .
\end{aligned}
$$

The solution of the problem is given by (32), which is general, together with (41), (50) and (51), which reflect the model assumed for $\varepsilon_{\mathrm{L}}$ and $\varepsilon_{\mathrm{T}}$. They could be derived in the same way for different models and then they would take different explicit forms with possibly more parameters and more subsidiary conditions. For sufficiently complicated dielectric functions it might even be necessary to resort to numerical evaluation of the integrals. But the point is made. A given model has been assumed and the problem has been completely and uniquely solved in terms of $p$ as a free parameter, without raising the question of A.B.C.

The inverse process described in $\S 2$ can now be demonstrated. Consider, for example, the case $p=1$. In this case (41), (50) and (51) yield easily $B_{0}=B_{3}=\xi=0$. Then (32) becomes

$$
\begin{aligned}
& i \kappa\left\{\int \exp (-i \lambda \eta)\left(-\frac{\kappa^{2}}{k^{2}}+\frac{\omega^{2}}{c^{2}} \frac{\lambda^{2}}{k^{2}\left(k^{2}-\frac{\omega^{2}}{c^{2}}\right)}\right) \mathrm{d} \lambda+\right. \\
& \left.\quad+\int \exp (i \lambda \eta)\left(-\frac{\kappa^{2}}{k^{2} \varepsilon_{\mathrm{L}}}+\frac{\omega^{2}}{c^{2}} \frac{\lambda^{2}}{k^{2}\left(k^{2}-\frac{\omega^{2}}{c^{2}} \varepsilon_{\mathrm{T}}\right)}\right) \mathrm{d} \lambda\right\}=0 .
\end{aligned}
$$

The first term is of course proportional to $\cos \theta$, which is the surface impedance of the vacuum. The surface impedance of the medium in question is written down at once from (4) and (9). This gives the factor needed to resolve the possible ambiguity discussed in $\S 2$. The factor is $4 \kappa / \omega$. In making this identification care must be taken about the sign convention, which depends on whether the medium for which $Z$ is being calculated is in $z>0$ or $z<0$. Finally, the surface impedance for P-mode and arbitrary $p$ is given by

$$
\begin{aligned}
i \pi \frac{\omega}{c}\left(1-\frac{c^{2} \kappa^{2}}{\omega^{2}}\right)^{1 / 2}-i & \frac{\omega}{4} Z_{\mathrm{P}}=\int\left\{\frac{\kappa^{2}\left(1+f_{1}\right)}{k^{2}}\left(\frac{1}{\varepsilon_{\mathrm{L}}}+1\right)+\right. \\
& \left.+\frac{i \kappa \xi\left(i v \varepsilon_{\mathrm{T}}+\lambda\right)}{\left(k^{2}-\frac{\omega^{2}}{c^{2}} \varepsilon_{\mathrm{T}}\right)}+\frac{\left(i \frac{\omega^{2}}{c^{2}} \lambda-v\right)\left(f_{2}+\frac{\lambda}{\kappa}\right) i \kappa}{\left(k^{2}-\frac{\omega^{2}}{c^{2}} \varepsilon_{\mathrm{T}}\right)}-\frac{\kappa^{2}\left(1+f_{1}\right)}{k^{2}}\right\} \exp (i \lambda \eta) \mathrm{d} \lambda .
\end{aligned}
$$

4. The quasi-static limit. - Having obtained the S.M.D.R. and the subsidiary conditions, different values of $p$ can now be used as desired. The most frequent ones are $p= \pm 1$, which have already been discussed, and $p=0$, which was used by Maradudin and Mills [2]. The connection with their work will be established in the quasi-static limit, $c \rightarrow \infty$, which is the case in which these authors give explicit results in final form. In this limit,

where

$$
r^{2} \rightarrow t^{2} ; \quad \kappa^{2}-R^{2} \rightarrow \frac{\omega^{2}}{c^{2}} \varepsilon
$$

$$
\varepsilon=\varepsilon(k=0, \omega)=\varepsilon_{\mathrm{c}}\left(1+\frac{\Omega_{\mathrm{p}}^{2}}{\omega_{\mathrm{T}}^{2}-\omega^{2}}\right)
$$

Then, from (51) in this limit,

$$
\xi=\frac{1}{\varepsilon(1+p)}\left((p-1)+i B_{0}\left(\frac{1}{l-\kappa}-\frac{p}{l+\kappa}\right)\right) .
$$

Hence, evaluating (20), the S.M.D.R. becomes

$$
(1+\varepsilon)\left\{\left(1+\frac{B_{0}}{i(l+\kappa)}\right) \frac{1}{\varepsilon}-\xi\right\}=\frac{\Omega_{\mathrm{p}}^{2}}{\left(\omega^{2}-\omega_{\mathrm{T}}^{2}\right)}\left\{\frac{1}{\varepsilon}\left(1+\frac{B_{0}}{i(L+l)}\right) \frac{1}{L}+\frac{\varepsilon_{\mathrm{c}} \xi}{t}\right\} \kappa,
$$


where $\xi$ and $B_{0}$ still depend on $p$. In particular, for $p=0,(56)$ and (41) yield

whence

$$
B_{0}=i(L-l) ; \quad \xi=-\frac{1}{\varepsilon} \frac{L-\kappa}{l-\kappa}
$$

$$
(1+\varepsilon)\left(\frac{(L+\kappa)}{(l+\kappa)}+\frac{(L-\kappa)}{(l-\kappa)}\right)=\frac{\Omega_{\mathrm{p}}^{2}}{\omega^{2}-\omega_{\mathrm{T}}^{2}}\left\{\frac{2}{L+l}-\varepsilon_{\mathrm{c}} \frac{(L-\kappa)}{(l-\kappa)} \frac{1}{t}\right\} \kappa .
$$

This gives the dispersion relation in analytic form for $p=0$ and arbitrary $\kappa$. The limit $\kappa \rightarrow 0$ can now be studied. This yields

$$
\omega^{2}=\omega_{0}^{2}+\frac{l_{0}}{2 L_{0}\left(1+\varepsilon_{\mathrm{c}}\right)} \Omega_{\mathrm{p}}^{2}\left\{\frac{2}{L_{0}+l_{0}}-\varepsilon_{\mathrm{c}} \frac{L_{0}}{l_{0}} \frac{1}{t_{0}}\right\} \kappa,
$$

where the subscript 0 indicates evaluation for $\kappa=0$. In particular

$$
\omega_{0}^{2}=\omega_{\mathrm{T}}^{2}+\frac{\varepsilon_{\mathrm{c}}}{1+\varepsilon_{\mathrm{c}}} \Omega_{\mathrm{p}}^{2} .
$$

The limit $\kappa \rightarrow 0$ for $l_{0}$ and $t_{0}$ needs some care, since there is the ambiguity that $\sqrt{-1}$ can be $\pm i$. Finally

$$
\omega^{2}=\omega_{0}^{2}+\Omega_{\mathrm{p}} \frac{\varepsilon_{\mathrm{c}}^{1 / 2}}{\left(1+\varepsilon_{\mathrm{c}}\right)^{3 / 2}}\left\{\varepsilon_{\mathrm{c}}^{1 / 2} \beta_{\mathrm{L}}-i \frac{\left(2 \beta_{\mathrm{L}}+\beta_{\mathrm{T}}\left(1+\varepsilon_{\mathrm{c}}\right)\right)}{2}\right\} \kappa,
$$

or, in terms of the static dielectric constant $\varepsilon_{\mathrm{s}}$,

$$
\omega=\omega_{0}+\frac{1}{2} \frac{\left(\varepsilon_{\mathrm{s}}-\varepsilon_{\mathrm{c}}\right)^{1 / 2}}{\left(1+\varepsilon_{\mathrm{c}}\right)^{3 / 2}} \frac{\omega_{\mathrm{T}}}{\omega_{0}}\left\{\beta_{\mathrm{L}} \varepsilon_{\mathrm{c}}^{1 / 2}-i \frac{\left(2 \beta_{\mathrm{L}}+\beta_{\mathrm{T}}\left(1+\varepsilon_{\mathrm{c}}\right)\right)}{2}\right\} \kappa .
$$

If $\beta_{\mathrm{L}}$ and $\beta_{\mathrm{T}}$ are assumed to be equal, then this gives the result obtained by Maradudin and Mills [2] for this particular case. It is important for the physical interpretation of the result to maintain the difference between these two terms because they have different origins and different physical roles. In order to make the discussion more complete it is convenient to write the long wave dispersion relation for arbitrary $p$. The result is

$$
\begin{aligned}
& \omega=\omega_{0}+\frac{(1+p)\left(\varepsilon_{\mathrm{s}}-\varepsilon_{\mathrm{c}}\right)^{1 / 2}}{2\left(1+\varepsilon_{\mathrm{c}}\right)^{1 / 2}} \frac{\omega_{\mathrm{T}}}{\omega_{0}} \times \\
& \times\left\{\frac{\varepsilon_{\mathrm{c}}^{1 / 2}(1+p) \beta_{\mathrm{L}}}{\left((1-p)^{2}+\varepsilon_{\mathrm{c}}(1+p)^{2}\right)}-i(1-p)\left(\frac{\beta_{\mathrm{L}}}{(1-p)^{2}+\varepsilon_{\mathrm{c}}(1+p)^{2}}+\frac{\beta_{\mathrm{T}}}{2(1+p)}\right)\right\} \kappa .
\end{aligned}
$$

Notice that for $p=1$ both contributions to the imaginary part vanish. This shows the dangers of drawing conclusions from comparing (63) with similar formulae often obtained in theories of surface plasmons for specular surfaces. The damping found in such cases has a different physical origin. It can be Landau damping, for example, which only appears if a dielectric function with appropriate structure is used. With simple minded functions such as the ones used in this paper the damping is zero for specular surfaces, in qualitative - but only qualitative - agreement with (64). Further contributions to the imaginary part arise if, for example, a finite surface potential barrier is used in the theory of surface plasmons, but this is also a different effect. Other and more important differences concerning free electron systems will be the subject of a separate publication.
Returning now to the systems studied in this paper, the field of the surface polariton for dispersive media contains an admixture of bulk polariton modes, as is seen from the poles in the upper half plane of the Fourier transform of the EM field in (25) through (28). This means a damping of the surface mode, via coupling to the bulk modes, as has been often pointed out by many authors. However, the present analysis shows that the strength of this coupling is zero for a specular surface model. Thus in this case this damping is absent. Furthermore, the really interesting term to look at is $\mathbf{H}_{g}^{\mathrm{M}}$ in (28). For finite $c$ the only poles are at $c^{2} k^{2}=\omega^{2} \varepsilon_{\mathrm{T}}$, which corresponds to the mixed - polariton - mode. In the quasi-static limit the poles are at $\varepsilon_{\mathrm{T}}^{-1}=0$ and this corresponds to the purely dynamical - exciton or plasmon - mode of the bulk system, uncoupled to the EM field $-g$ is 
of order $c / \omega$. This is the only term which contributes to the damping of the surface mode in this limit. Its contribution for long waves appears in the term in $\beta_{\mathrm{T}}$ in (64), which displays the factor $(1-p)$ explicitly. The other imaginary term in $\beta_{\mathrm{L}}$ does not correspond to surface-bulk coupling. It has been shown by Flores and García-Moliner [17] that the S.M.D.R. in the quasi-static limit is determined quite generally by two integrals. One is associated with the fluctuations of $E_{z}(z)$ and is of no interest for the present discussion. The other one is

$$
\int_{0}^{\infty} \frac{D_{x}(z)}{E_{x}(\infty)} \mathrm{d} z
$$

In the long wave limit this gives a contribution to the coefficient of $\kappa$ in the dispersion relation. This term represents the role of current density fluctuations displacement current, in the case of dielectrics parallel to the surface. Except for $p=1$, this term decays via surface scattering. Its contribution to the imaginary part in (64) is the term in $\beta_{\mathrm{L}}$, which of course has also the factor $(1-p)$. Thus, although both terms add up in (64) and (63), the assumption that $\beta_{\mathrm{T}}=\beta_{\mathrm{L}}$, even if it is numerically reasonable, may lead to an erroneous physical interpretation of the result.

5. Conclusions. - Maxwell's equations and the standard matching conditions imposing the correct behaviour of the EM field across the boundary do not contain sufficient information to solve the problems of surface electrodynamics of dispersive media. It is necessary to specify a model and this includes the complete dielectric tensor of the bulk medium and the effect of the surface on the scattering of the excitations-polaritons. This paper gives the complete solution in terms of a phenomenological model of surface scattering using a specularity parameter $p$. This is only an approximate way of describing surface scattering, but real progress beyond this point has yet to be made. For the time being it is sufficiently interesting to solve the phenomenological model for any arbitrary value of $p$ and this is what has been done here.

An alternative approach consists in seeking additional boundary conditions. This can be done, but the analysis in terms of the broad model carried out in this paper shows that the different forms of A.B.C. which have been used are the result of some specific model, corresponding to particular values of $p$. This emphasizes that it would be erroneous to conclude that the non local form of Maxwell's equations is sufficient to determine the A.B.C. Indeed there seems to be no obvious advantage in this approach. It amounts to an extra formal complication and, by implicitly depending on assumptions based after all on some model, it may lead to erroneous generalizations. It seems more physical to proceed directly from a given model, which can be prescribed in a fairly broad way, and to keep track of the different terms. This makes possible a direct physical interpretation of the results. Moreover, the problem has been solved here for arbitrary $p\left({ }^{1}\right)$.

It may be in order to discuss further the meaning of the parameter $p$. At the phenomenological level its meaning is expressed by eq. (2) of $\S 1$ and eqs. (19-21) of $\S 3$ or, alternatively by the way of writing a formula for the susceptibility in the manner of Birman and associates [9,10], as recalled here in $\S 1$. To go beyond this requires the introduction of a model. The easiest case to visualize is that of an ionic vibrating lattice the fact that an actual lattice may require more elaborate dielectric functions than the simple ones used here means only that one should not expect explicit results based on (34) and (36) to be very reliable beyond relatively long wavelengths but this is a separate issue which does not affect the argument concerning the boundary conditions or the role of surface scattering. The situation may be physically envisaged as follows : when the wave as a travelling excitation reaches the atoms at the surface, these in turn vibrate and thus reemit the wave inwards. Since the interaction constants, as well as other conceivable constraints on the vibrating atoms, are in general different at the surface, even if the surface is otherwise nearby perfect one would expect the wave to be reemitted with some phase lag, due to the fact that the vibrational response of the atoms at the surface is different from that of the atoms in the bulk. One would then expect the parameter $p$, which weighs the reflected wave component, to be of the form $p=\exp (i \varphi)$. Then, when the response of the surface atoms is equal to that of the bulk atoms one would be in the ideal specular case $\varphi=0$, whereas in the opposite extreme $(\varphi=\pi)$ the surface atoms are totally restricted, i.e., they cannot vibrate. This corresponds to the situation of anti-specular scattering. With $p=-1$ the combination of incident and reflected wave gives zero amplitude for $z=+0$. In support of this view one can consider the following argument, which incidentally gives further insight into the relationship between surface scattering model and A.B.C.

From the solution obtained in this paper one can calculate the fields $\mathbf{E}$ and $\mathbf{D}$ everywhere inside the material. Thus one can write down $\mathbf{P}$ as a function of $z$ and hence $\partial P / \partial z$. Let us concentrate on P-mode geometry and on the $x$-component.

Furthermore, to simplify the argument let us take $\beta_{\mathrm{L}}^{2}=\beta_{\mathrm{T}}^{2}=D$, as in [2]. We can then evaluate $P_{x}$ and $\partial P / \partial z$ at $z=+0$ and find

$$
\left[P_{x}+b \frac{\partial P_{x}}{\partial z}\right]_{+0}=0 ; \quad b=\frac{i}{\Gamma} \frac{(p+1)}{(p-1)}
$$

( $\left.{ }^{1}\right)$ While this paper was in preparation a recent publication [19] came to our attention in which a single solution has been obtained which is valid for the two cases $p= \pm 1$. 
where $\Gamma$, introduced in [2], is a real quantity for frequencies in the range of interest. The form of (65) is that in which it has been customary to cast the A.B.C. We see here the explicit connection between this and the parameter $p$ without resorting to extreme cases. Now, Bishop and Maradudin [18] have recently discussed the problem of energy conservation - for the phonon case - in relation to the conditions at the surface and shown that in the case of normal incidence $b$ must be real in order to ensure the conservation of energy. Our intuitive picture of surface reflection leads to precisely this, i.e., for $p=\exp (i \varphi)$ and $\Gamma$ real $b$ is just real - in this discussion we are ignoring possible bulk dissipative mechanisms, which constitute a different issue. With this proviso $\Gamma$ is real for the frequency range of interest. The case $p=0$, which corresponds to the A.B.C. (3.22) of [2], has been discussed above only to see in explicit terms the connection with the results of Maradudin and Mills. Of course, as shown by Bishop and Maradudin [18] this would not of itself conserve energy, so that in order to provide a physical basis for the model $p=0$ one would have to think of some appropriate external force introducing an energy source - or sink -, but the point of this discussion is to explain what $p$ means. A different problem is to produce a physical model for which $p$ is evaluated.

In the above discussion we have resorted to a picture in terms of phonons but, as emphasized by Hopfield and Thomas [18], the excitations which carry dielectric polarization can be conceived in abstract form. Excitons are often the object of interest and a similar picture could be built up for this case. In fact eq. (14) of [8] corresponds precisely to a reflection parameter of the form $\exp (\mathrm{i} \varphi)$ whose calculation evidently would require a macroscopic understanding of the forces which cause the exciton to turn around. An explicit model for excitons, after Pekar's suggestion [6], assumes that the polarization associated with the exciton is zero at $z=+0$, i.e., that $p=-1$. However - see discussion at the end of [7] - also for excitons this is only an extreme case.

Now, all the preceeding discussion is based on the concept of a wave, in which the phase is a meaningful concept. The situation is different in a conductor, where the particles undergoing surface scattering are charge carriers. In a semi-classical picture all that matter is the number of carriers reaching and leaving the surface. The parameter $p$ in this case counts the real fraction of specularly scattered carriers and corresponds to the parameter used in Boltzmann equation formulations of surface transport. This problem will be fully discussed in a forthcoming publication. It will suffice here to stress two points, namely : (i) For conductors $p$ takes physically meaningful values in the real interval $(0,1)$, and (ii) given $p$, the method used here to solve the surface problem is not valid for conductors. The reason is that in a conductor for any values of $p \neq 1$ this model would describe an unphysical accumulation of charge at the surface. This is most clearly seen in the case $p=0$. In this case the model, as developed here, would not provide any means for the return back from the surface into the bulk of the current coming from the bulk. Such a problem of charge accumulation does not arise with dielectrics. Conducting surfaces require a different treatment and this will be done in the following publication.

\section{References}

[1] Agranovich, V. M. and GinzbURG, V. L., Spatial dispersion in crystal optics and the theory of excitons (Interscience, London) 1966.

[2] Maradudin, A. A. and Mills, D. L., Phys. Rev. B 7 (1973) 2787.

[3] Lagois, J. and Fischer, B., Solid State Commun. 18 (1976) 1519.

[4] Rimbey, P. R. and Mahan, G. D., Solid State Commun. 15 (1974) 35.

[5] Mills, D. L., Polaritons (Proceedings 1st Taormina Research Conference on Structure of Matter 1972) E. Burstein and F. de Martini (Eds.) (Pergamon Press, N.Y.) 1974.

[6] Pekar, S. I., Sov. Phys. J.E.P.T. (A.I.P.) 6 (1958) 785.

[7] Hopfield, J. J., J. Phys. Soc. Japan 21 (Suppl.) (1966) 77.

[8] Hopfield, J. J. and Thomas, D. G., Phys. Rev. 132 (1963) 563.

[9] Birman, J. L. and ZeYher, R., Polaritons (Proceedings 1st Taormina Research Conference on Structure of Matter 1972) E. Burstein and F. de Martini (Eds.) (Pergamon, N.Y.) 1974.
[10] Frankel, M. J. and Birman, J. L., Phys. Rev. B 13 (1976) 2587.

[11] Skettrup, T., Phys. Status Solidi (b) 60 (1973) 695.

[12] AKHIEZER, N. I., The calculus of variations (Blaisdell, N.Y.) 1962.

[13] Kliewer, K. L. and Fuchs, R., Phys. Rev. 172 (1968) 607.

[14] Mahan, G. D., Elementary excitations in solids, molecules and atoms Part B, J. T. Devreese, A. B. Kunz and T. C. Collins (Eds.) (Plenum Press, London \& N.Y.) 1974.

[15] Lipson, S. G. and Lipson, H., Optical Physics (Cambridge Univ. Press) 1969.

[16] Flores, F. and Navascues, G., Surf. Sci. 34 (1973) 773.

[17] Flores, F. and GarCía-Moliner, F., Solid State Commun. 11 (1972) 1295.

[18] Bishop, M. F. and Maradudin, A. A., Phys. Rev. B 14 (1976) 3384.

[19] Johnson, D. L. and Rimbey, P. R., Phys. Rev. B 14 (1976) 2398. 